null • Vol. 2, No. 8

\title{
Review: Gulu SoundTracks
}

Damascus Kafumbe ${ }^{1}$

${ }^{1}$ Middlebury College

Published on: Aug 16, 2021

License: Creative Commons Attribution 4.0 International License (CC-BY 4.0). 


\section{Project}

Gulu SoundTracks

Project Director

Joella Bitter, Duke University

Project URL

http://gulusoundtracks.org/

Project Reviewer

Damascus Kafumbe, Middlebury College

\section{Project Overview}

\section{Joella Bitter}

Gulu SoundTracks curates a collection of eight audio tracks as a digital ethnographic sound project. The project invites listeners into the sonic habitus and imaginations of their Gulu-based creators. As a collection, the album offers a series of stories inspired by multiple histories of listening to a place and recording its sounds. These are narratives evoked musically - without lyrics - through the aesthetic manipulations of densely-layered "soundscape" audio samples, software instrumental sounds, and cyclical grooves. The compilation innovates on the ethnographic genre by blending multiple methods and forms through the affordances of the digital. Drawing inspiration from the ethnomusicological album (e.g., Smithsonian Folkways or Gruenrekorder) and multi-authored forms of scholarship (e.g., edited volumes), this project poses one possibility for a more-public sounded anthropology.

The process of creating Gulu SoundTracks happened in four stages: field recording (2014-2018), sound production (2018), the design and construction of the digital site (2019-2020), and audience engagement (2020-as long as the site exists). Our team grew with each stage of the project. I (Bitter) completed all field audio recording and compiled a selection of audio samples into a digital folder distributed to each of the studios. Music producers Black da Massacre, Kaunda, Mellix, and Usaih then each composed, produced, and narrated the audio tracks using selections of this audio. Back in Durham, Liz Milewicz and Will Shaw at Duke University's Digital Scholarship Services provided expertise on project planning and dissemination. From other corners of the Duke Library System, Paolo Mangiafico contributed communications expertise, 
Arnetta Girardeau provided copyright expertise, and Maggie Dickenson consulted on metadata construction. Finally, the web design for the project was completed in collaboration with visual artist Clara Pinchbeck. As part of the web design process, I prepared text and created a series of collages using photographs taken in Gulu by photographer Ojok Eddie and me.

Gulu SoundTracks was designed with academic and lay audiences in mind. For those interested foremost in the tracks as listening artifacts, the audio can be streamed or downloaded, either individually or as an album. For those interested in an explication of the tracks' ethnographic-imaginative storytelling, the "Liner Notes" offer textual synopses and a more audiovisual experience. For those interested in process and its analytic underpinnings, the "Project + Process" and "Acknowledgements +" pages provide an overview. And, for those interested in learning more about Kaunda, Usaih, Black, and Mellix, the "Contributors" page provides brief biographies and links to their music. These design choices reflect an interest in conversations about experimental ethnography, multimodality, and accessibility.

The recording and production stages of this project received support from the WennerGren Foundation and National Science Foundation as part of my dissertation research. The web design stage received support from the Mellon-funded Ethnography Workshop at Duke University. A presentation explaining learning points in the effort to create an internationally-accessible digital sound scholarship project was given at Duke Library's 2020 Digital Scholarship Open House. In September 2020, Gulu SoundTracks received media coverage from Duke's Graduate School.

\section{Project Review}

\section{Damascus Kafumbe}

Gulu SoundTracks: Remixing the Storied City uses eight instrumental audio tracks, collages, and text to construct a digital ethnographic account of everyday life in the Ugandan city of Gulu. The eight tracks serve as sonic stories, expressions, and markers of Gulu's sociocultural space and its soundscapes. These instrumentals are original productions of four local music producers, who use digital music software and draw on various processes-layering, sampling, and looping, among others. The tracks draw on and translate local and foreign music as well as dance idioms and styles. In addition, they feature a wide range of city-sounds that Joella Bitter, a U.S.-based anthropologist and artist, recorded during her doctoral ethnographic research in Gulu 
between 2014 and 2018. The instrumentals' corresponding collages are Bitter's original creations, consisting of photographs of Gulu that she and a local collaborator took during her fieldwork there. In the project's accompanying text, the collaborators describe the project's liner notes as "textual derivations of storied concepts narrated by each producer, in conversation with the group, during... [their] collective post-production listening session in Gulu in 2018." However, it is unclear from the notes if their collaboration occurred in English, in Gulu's staple language, Acholi, or in both.

Gulu SoundTracks is a unique collaborative and accessible digital humanities project. It blurs the line between ethnographer and interlocutor by distributing credit evenly among collaborators while highlighting the unique contributions of each. The project allows local participants an active say in how it documents, represents, and conceptualizes their sociocultural space by bringing the soundscapes of the city to life through the creative work and perspectives of five of its residents. Moreover, the project's localization on a website makes it more accessible to the general public, though it is still restricted to those who have internet access. All the same, the versatility of the website is fundamental to the functionality and reception of the project. For instance, its dynamic and multi-media format allows it to present "remixed" musical compositions as a form of ethnography. It also allows the works and their accompanying collages to function as artistic reimaginings of Gulu's sociocultural space. Moreover, the structure and format of Gulu SoundTracks's website remains in theme with the philosophy of "remixing" because it "remixes" prior ethnographic work to fit new needs.

The creators of Gulu SoundTracks challenge traditional approaches to ethnographic work by demonstrating the potential posed by the "intersections of experimental ethnography, creative arts, digital humanities, and sound studies," among other fields. The collaborators might broaden the project's appeal by problematizing the implications that such an "experimental" project might have for varied audiences, including the broader Gulu community and other ethnographic researchers. Similarly, they might augment the project's local relevance by contextualizing the idea of "remixing" in various narratives of the Acholi community of northern Uganda. Gulu SoundTracks is a vital intervention in contemporary ethnographic practice, as well as arts and humanities scholarship. It makes use of its dynamic medium by expressing the complexity of Gulu's sociocultural space and posing as an interface through which we might encounter and engage with that space further. 